\title{
Hypoxic Preconditioning Attenuated Simulated Ischemia/Reperfusion Injury in Cultured Bone Marrow Stromal Cells
}

\author{
LiHua Shen ${ }^{1 *}$, ErBing Wu${ }^{1}$, Lin Cao ${ }^{1}$, Jin Chen ${ }^{1}$, Cuiping Xia1, Yu Long ${ }^{1}$ and Xinsheng Ding ${ }^{2 *}$ \\ ${ }^{1}$ Department of Neurology, The Affiliated Hospital of Nantong University, 20 XiSi Road, Nantong, 226001, P.R.China \\ ${ }^{2}$ Department of Neurology, The First Affiliated Hospital of Nanjing Medical University, 300 GuangZhou Road, Nanjing, 210029, P.R.China
}

\begin{abstract}
Aim: Bone marrow stromal cells (BMSCs) are a promising candidate for cell therapy in ischemic stroke. However, the majority of BMSCs are readily lost after transplantation because of apoptosis due to ischemia-reperfusion (I/R) injury. In the present study, we aimed to evaluate the effects of hypoxia preconditioning (HP) on simulated I/R injury in cultured BMSCs.

Methods: Four generations of BMSCs were used as subjects. BMSCs were divided into 6 groups, including normal group, I/R group(I/R followed $0 \mathrm{~h} \mathrm{HP}), 2 \mathrm{~h}-\mathrm{HP}$ group (I/R followed $2 \mathrm{~h} \mathrm{HP}), 4 \mathrm{~h}-\mathrm{HP}$ group (I/R followed $4 \mathrm{~h} \mathrm{HP})$, $6 \mathrm{~h}-\mathrm{HP}$ group (I/R followed $6 \mathrm{~h} \mathrm{HP}$ ) and $8 \mathrm{~h}-\mathrm{HP}$ group(I/R followed $8 \mathrm{~h} \mathrm{HP}) 2 \mathrm{~h}-\mathrm{HP}$ group(l/R followed $2 \mathrm{~h} \mathrm{HP}$ ), $4 \mathrm{~h}-\mathrm{HP}$ group(I/R followed $4 \mathrm{~h} \mathrm{HP}$ ), $6 \mathrm{~h}$-HP group (I/R followed $6 \mathrm{~h} \mathrm{HP}$ ) and $8 \mathrm{~h}-\mathrm{HP}$ group(I/R followed $8 \mathrm{~h} \mathrm{HP})$. BMSCs were subjected to HP by exposing the cells to hypoxia $\left(2 \% \mathrm{O}_{2}\right)$. After $12 \mathrm{~h}$ of reoxygenation, BMSCs were suffered from $3 \mathrm{~h}$ ischemia $\left(<0.5 \% \mathrm{O}_{2}\right.$ and serum deprivation) and subsequent reperfusion (I/R). Cell viability, hypoxia-inducible factor (HIF) 1-a, caspase-3 and apoptosis were tested using MTT, ELISA and immunofluorescence staining respectively.

Results: There were no changes in cell viability after $2 \mathrm{~h}$ to $8 \mathrm{~h}$ HP in cultured BMSCs as compared to the normal group. But HP upregulated the content of HIF-1 $\alpha$ in BMSCs and protected the BMSCs from later I/R injury, as evidenced by increased cell viability and decreased Caspase- 3 release and apoptosis, especially in $8 \mathrm{~h}-\mathrm{HP}$ group

Conclusion: HP attenuated the apoptosis induced by I/R injury via co-regulating the expression of HIF-1 $\alpha$ and Caspase-3 in BMSCs, and thereby might play a beneficial effect on cell therapy.
\end{abstract}

Keywords: Hypoxic preconditioning (HP); Cell viability; Hypoxiainducible factor (HIF) 1- $\alpha$; Apoptosis; Ischemia/reperfusion (I/R) injury

Abbreviations: ANOVA: analysis of variance; BMSCs: Bone marrow stromal cells; ELISA: enzyme-linked immunosorbent assay; HP: hypoxia preconditioning; HIF: hypoxia-inducible factor; I/R: ischemia-reperfusion; PBS: phosphate buffered solution

\section{Introduction}

Ischemic stroke is one of the leading causes of death and disability in the world, but there are currently few effective clinical therapies for it. Alternatively, cell-based therapy has provided a promising hope to enhance tissue repair and functional recovery after stroke [1-3]. Bone marrow stromal cells (BMSCs) can be easily obtained from patients themselves without ethical or immunological problems and can proliferate massively in vitro. After transplantation, its can promote the neurogenesis and angiogenesis in ischemic brain [4]. However, the cell therapy is limited by the poor survival of the transplanted cells in brain lesion [5]. Studies confirmed that a large number of transplanted cells died in ischemic brain because of hypoxia, oxidative stress, inflammatory mediators and the deficiency of trophic factor [6,7]. Thus, it is imperative to identify therapies that can improve the viability of the stem cells in the hazardous ischemic tissue. The physiological oxygenated environment for BMSCs in bone marrow not exposed to atmospheric oxygen is thought to range from $2-8 \%$ [8]. Recent study showed hypoxic preconditioning(HP) could decrease apoptosis induced by anoxia in cultured hippocampus neurons [9]. So in this study, we wanted to study whether HP might attenuate the apoptosis of BMSCs from stimulated ischemic/reperfusion (I/R) injury. We elaborated cell viability, hypoxia-inducible factor (HIF) $1-\alpha$, apoptosis and Caspase- 3 appreciate in this protection mechanism. Furthermore, the proper time of HP involved was also studied.

\section{Materials and Methods}

\section{Isolation and culture of BMSCs}

BMSCs were isolated as previously described (Deng et al., 2004) [10]. Briefly, we humanely killed male Sprague-Dawley rats (3-4 weeks old), bone marrow was obtained from the rat femurs and tibias. Cells were centrifuged at $1000 \mathrm{rpm}$ for $5 \mathrm{~min}$ and suspended in Dulbecco's modified Eagle medium with low glucose (L-DMEM, Invitrogen, Carlsbad, CA) supplemented with $12.5 \% \%$ fetal bovine serum (FBS, Invitrogen, Carlsbad, CA). Cell cultures were kept in humidified $95 \%$ air- $5 \% \mathrm{CO}_{2}$ at $37^{\circ} \mathrm{C}$. After the cells were incubated for $3 \mathrm{~d}$, the culture medium was changed. Once the BMSCs reached $80 \%$ confluence, the adherent cells were digested using $0.25 \%$ trypsin with EDTA. At passage 4 (P4), cells were harvested for experiments.

*Corresponding author: LiHua Shen, The Affiliated Hospital of Nantong University, 20 XiSi Road, Nantong, 226001, P.R.China, Fax: +86 51385519820 E-mail: Lihuashen139@yeah.net

Xinsheng Ding, Department of Neurology, The First Affiliated Hospital of Nanjing Medical University, 300 GuangZhou Road, Nanjing, 210029, P.R.China, Tel: 8613951972060; E-mail: 13951972060@139.com

Received November 18, 2013; Accepted February 24, 2014; Published February 24,2014

Citation: Shen L, Wu E, Cao L, Chen J, Xia C, et al. (2014) Hypoxic Preconditioning Attenuated Simulated Ischemia/Reperfusion Injury in Cultured Bone Marrow Stromal Cells. J Cytol Histol 5: 222. doi:10.4172/2157 7099.1000222

Copyright: (C) 2014 Shen L, et al. This is an open-access article distributed under the terms of the Creative Commons Attribution License, which permits unrestricted use, distribution, and reproduction in any medium, provided the original author and source are credited. 


\section{Hypoxic Preconditioning (HP) and experimental protocols}

Cultured BMSCs were divided into 6 groups(n=5/group): (1) the normal control group, BMSCs were placed in normal culture condition $\left(21 \% \mathrm{O}_{2}\right.$ and normal cultured medium) during the all experiment; (2) I/R group: BMSCs were cultured in L-DMEM without FBS and exposed to hypoxia for $3 \mathrm{~h}\left(<0.5 \% \mathrm{O}_{2}\right)$ in an airtight chamber (NAPCO $7101 \mathrm{FC}-1)$, followed by $24 \mathrm{~h}$ of reoxygenation $\left(21 \% \mathrm{O}_{2}\right.$ and normal cultured medium). There was no HP treatment before I/R injury. (3) 2 h-HP group: 2 hours HP treatment before I/R injury. (4) 4 h-HP group, 4 hours HP treatment before I/R injury. (5) 6 h-HP group: 6 hours HP treatment before I/R injury. (6) 8 h-HP group: 8 hours HP treatment before I/R injury. BMSCs were subjected to HP by exposing the cells to hypoxia $\left(2 \% \mathrm{O}_{2}\right)$, after $12 \mathrm{~h}$ of reoxygenation, BMSCs were suffered from $3 \mathrm{~h} \mathrm{I} / \mathrm{R}$ injury. The oxygen level in the chamber was monitored with an oxygen analyzer; some assays were carried out immediately after HP, others assays were administered followed the I/R attack. We use a graphical to depict our study in briefly (Figure 1).

\section{Cell viability assay}

The cells were seeded in 96-well plates at a density of $5 \times 10^{4}$ cells/ well at passage 4 . After HP and I/R procedure, the media was replaced with 3-(4,5-methylthiazol-2-yl)-2,5-diphenyl-tetrazolium bromide (MTT)(Sigma-Aldrich, USA)-containing DMEM, and cultured for 4 $\mathrm{h}$ in a $\mathrm{CO}_{2}$ incubator respectively. An equal volume of solubilization solution ( $10 \%$ SDS, $0.01 \mathrm{M} \mathrm{HCl}$ ) was added, and the plate was incubated at $37^{\circ} \mathrm{C}$ overnight to solubilize formazon crystals. When dissolved in SDS, functional mitochondrial succinate dehydrogenase in cells can convert MTT to formazan that generates a blue color. After the purple formazan producer was dissolved, the intensity was measured at an absorption wave length of $570 \mathrm{~nm}$ and $630 \mathrm{~nm}$ with a microplate reader.

\section{ELISA for HIF-1 $\alpha$ and Caspase-3}

BMSCs in 6-well plates were covered with $2 \mathrm{ml}$ of culture medium and experienced $2 \mathrm{~h}, 4 \mathrm{~h}, 6 \mathrm{~h}$ and $8 \mathrm{~h} \mathrm{HP}$ respectively, culture medium were withdrawn after HP, HIF-1 1 levels were measured in these samples with an ELISA kit for rat HIF-1 $\alpha$ (CUSABIO, China) according to the manufacturer's instructions. The protein extract was obtained from the supernatant of BMSCs.

\section{Hoechst 33342 fluorescent stain}

When cells sufficiently spread on the slides, the cells were hed with cold phosphate buffer sodium (PBS, pH 7.0) solution and fixed in $4 \%$ methanol for $30 \mathrm{~min}$. After washing with PBS for $5 \mathrm{~min}$, the cells were incubated with $0.1 \%$ acetic acid for $30 \mathrm{~s}$, and then washed again with PBS. Cells were thoroughly air-dried at room temperature, and then stained with Hoechst 33342 for 10 min, followed by washing with distilled water for $1 \mathrm{~min}$ and again air-drying at room temperature. Finally, the BMSCs were observed under fluorescence microscope and the apoptotic cells were photographed.

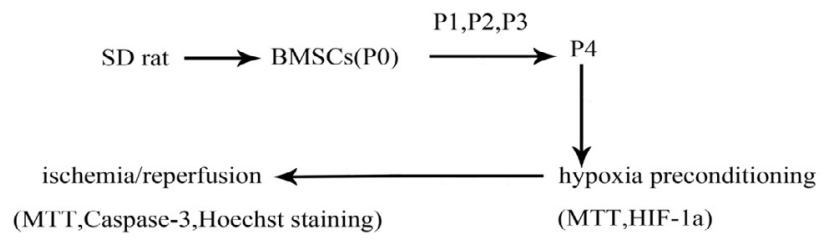

Figure 1: Experiment procedure.
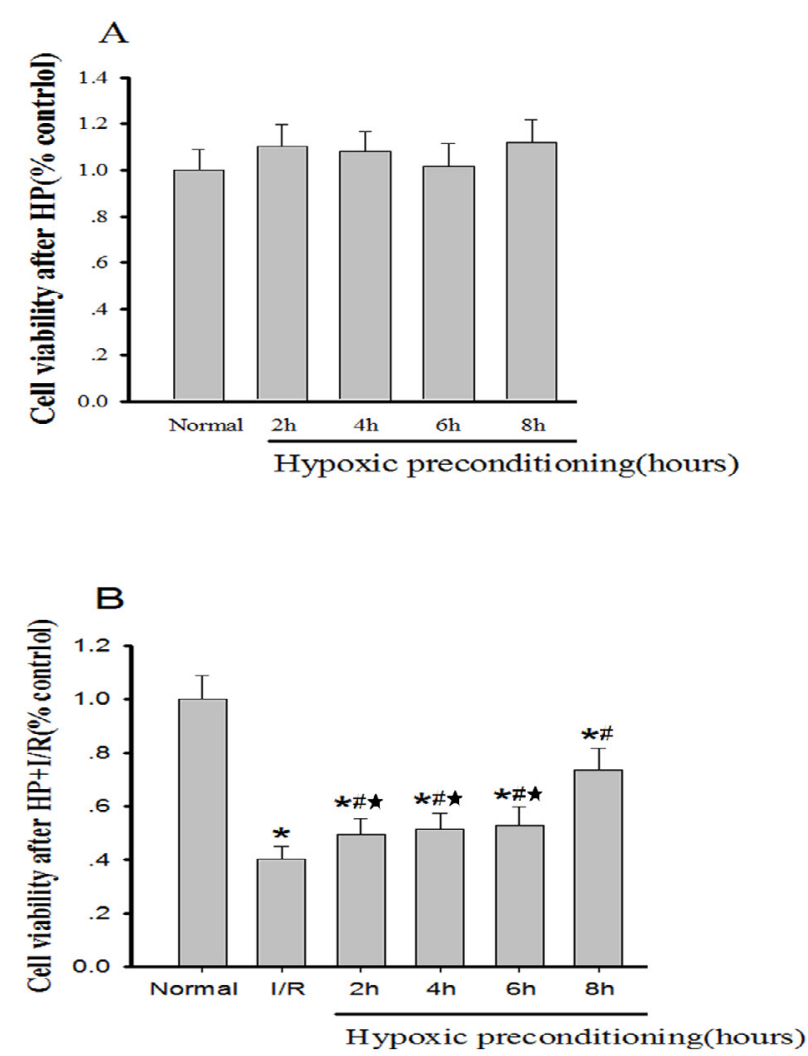

Figure 2: Cell viability measured by MTT assay. (A): Cell viability measured after HP, there was no statistic difference among all groups. (B): Cell viability measured after I/R injury, $Y$ axis value $=O D$ value $(570-630 \mathrm{~nm})(\mathrm{HP}$ groups $) / O D$ value $(570-630 \mathrm{~nm})$ (normal group)\%, ${ }^{*} p<0.01$, vs. normal group , $\# p<0.01$, vs. I/R group, * $\mathrm{p}<0.01$, vs. 8 h HP group.

\section{Statistical analysis}

All measurements were performed blindly. Results were presented as mean \pm SD. Statistical analyses were performed using SPSS 17.0 software. The statistical differences between different groups were evaluated with one-way analysis of variance (ANOVA) and post hoc test LSD (Fisher's Least Significant Differences) for multiple comparisons. A value of $\mathrm{P}<0.05$ was considered statistically significant.

\section{Results}

\section{Effect of HP on BMSCs viability}

There were no statistical differences in the cell viability among the normal culture and HP treated BMSCs groups (Figure 2A). Increasing exposure to $2 \% \mathrm{O}_{2}$ for 12 to $24 \mathrm{~h}$ resulted in duration-dependent apoptosis (data are not shown here). This suggested that BMSCs exposed to hypoxia from $2 \mathrm{~h}$ to $8 \mathrm{~h}$ remained fully viable similar to normoxia control BMSCs. But cell viability was decreased after subsequent I/R ( $<0.5 \% \mathrm{O}_{2}-3 \mathrm{~h}$, without $\mathrm{FBS}$, followed by reoxygenation) injury. However, HP treated BMSCs reduced the injury, especially $8 \mathrm{~h}$ $\mathrm{HP}$ before I/R insult presented an outstanding protection (Figure $2 \mathrm{~B}$ ).

\section{HP induced additional HIF-1a expression in BMSCs}

To further understand the mechanism of the protection of HP on anoxia, we tested the HIF-1 $\alpha$ expressions in normal and HP treated BMSCs. We found the contents of HIF-1a, a survival factors for some 
cell lines in response to hypoxia, in the BMSCs was significantly upregulated after $\mathrm{HP}(\mathrm{P}<0.05)$, and $8 \mathrm{~h}-\mathrm{HP}$ showed a prominent role $(\mathrm{P}<0.01)$ (Figure 3$)$.

\section{HP inhibited the activation of Caspase-3}

Caspase- 3 is released by damaged degenerating cells and plays a key role in the mechanism of apoptosis. Here we tested the production of activated caspase-3 in BMSCs. As shown in Figure 4, the contents of activated caspase- 3 were obviously increased in I/R group, but HP powerfully inhibited the harmful reaction $(\mathrm{P}<0.05)$. Similarly, $8 \mathrm{~h}-\mathrm{HP}$ showed a significant antiapoptosis role $(\mathrm{P}<0.01$, vs. I/R group).

\section{HP decreased the expression of apoptotic body}

Hoechst 33342 staining was used to observe apoptotic body. Only a few cells in normal culture group showed positive staining in the nuclei(apoptotic cell), while as a large number of cells in I/R group exhibited strong staining in the nuclei, but the apoptotic density was obviously decreased in 8 h-HP I/R injury group (Figure 5).

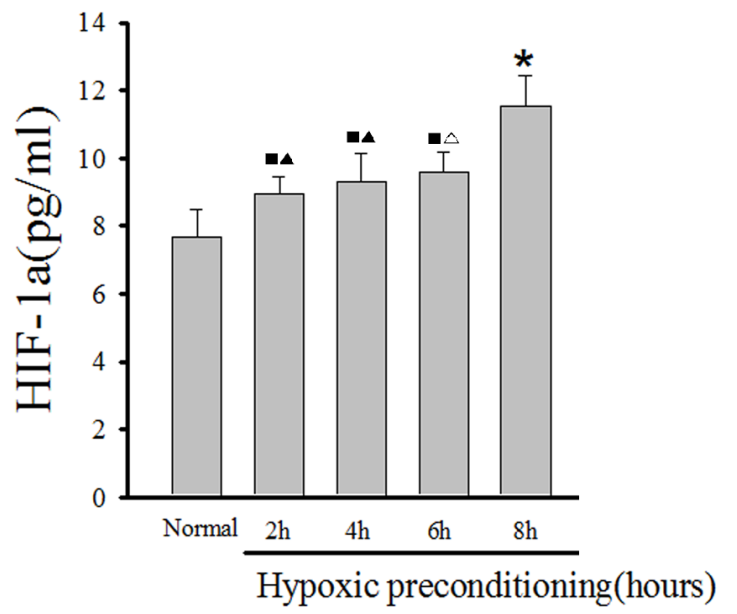

Figure 3: The content of HIF-1a in cultured BMSCs after I/R injury. " $p<0.05$, vs. normal group, ${ }^{*} p<0.01$, vs. normal group, ${ }^{\star} p<0.01$, vs. 8 h HP group, ${ }^{\Delta} p<0.05$ vs. $8 \mathrm{~h}$ HP group

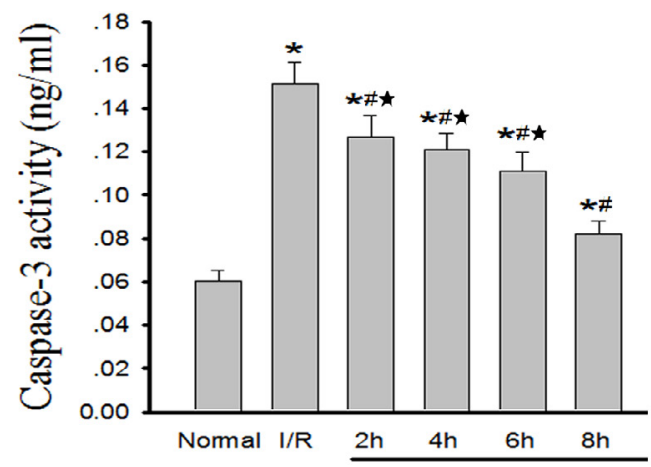

Hypoxic preconditioning(hours)

Figure 4: The content of active Caspase-3 in cultured BMSCs after I/R injury. ${ }^{*} p<0.01$, vs. normal group, ${ }^{\#} p<0.01$, vs. I/R group, ${ }^{\Delta} p<0.01$, vs. 8 h HP group.
A
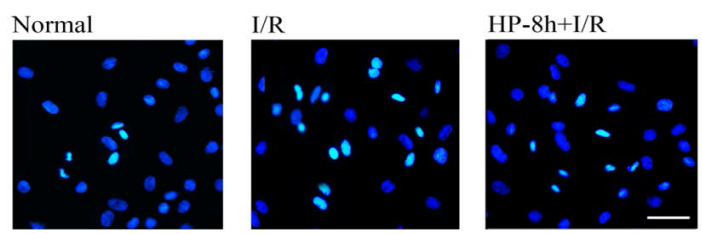

B

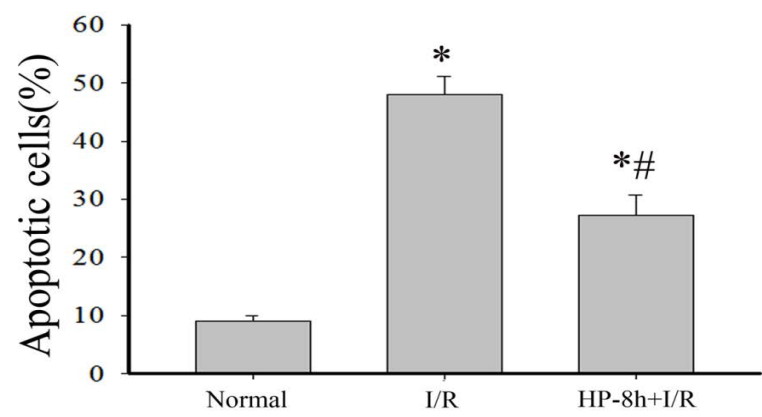

Figure 5: Influence of HP on BMSCs apoptosis after I/R injury. A: Hoechs 33342 staining in the cultured BMSCs, the strong staining nucleus represent the apoptosis cells. B: The percent of apoptotic cells in normal group, I/R injury without HP group and I/R injury with $8 \mathrm{~h}$-HP group. ${ }^{*} \mathrm{p}<0.01 \mathrm{vs.} \mathrm{normal} \mathrm{group,}$ ${ }^{\#} p<0.01$ vs. I/R group. scale bar indicates $200 \mu \mathrm{m}$.

\section{Discussion}

Transplantation of BMSCs is a potential therapy for ischemic stroke, however, a constant outcome that dramatically impairs the efficacy of the cell therapy is the limited number of stem cells surviving after graft in brain lesion $[11,12]$. Thus the valid defensive measures have been sought to prevent the engrafted cells from ischemic injury for a long time. A large number of data have been reported that the application of brief, transient periods of non-lethal hypoxia (hypoxic preconditioning, $\mathrm{HP}$ ) before a subsequent lethal episode of hypoxia markedly increase the endurance against followed severe anoxia and delay the development of programmed cell death [13-15]. Thus, HP might provide a new and hopeful strategy for cell therapy in ischemic diseases through the transformation of gene expression and activation of intracellular signaling pathways [16-20]. In vitro experiment, HP protected the myocytes from succedent hypoxia/reoxygenation injury by decreased apoptosis and LDH release and increased cell viability [21]. In vivo, HP was shown to be neuroprotective against ischemic brain injury through the upregulation of pro-survival and endogenous regeneration [22,23]. In the present study, we firstly simulated the physiological oxygenated tension $\left(2 \% \mathrm{O}_{2}\right)$ of BMSCs as the HP condition in cultured BMSCs and analysed the optimal time duration and mechanism of HP in reducing $\mathrm{I} / \mathrm{R}$ injury. We found $2 \mathrm{~h}$ to $8 \mathrm{~h} \mathrm{HP}$ did not arouse any harmful effect on cell viability. In addition, the contents of HIF-1a in BMSCs were up-regulated followed HP, when cells subsequently suffered from I/R injury, the decreased caspase- 3 release and increased cell viability were seen in HP groups as compared to I/R without HP group.

HIF-1a is a transcription factor specifically activated by hypoxia, in proper low oxygen pressures, the level of HIF-1 a is regulated to maximal involving multiple mechanisms of control at the levels of mRNA expression, protein stability, nuclear translocation and transactivation activity [24], and play a protective role in the antiapoptosis after ischemia $[25,26]$. Caspase- 3 , one member of the caspase family, is in the downstream of Bax, and particularly believes to be most commonly involved in the execution of apoptosis induced by 
Citation: Shen L, Wu E, Cao L, Chen J, Xia C, et al. (2014) Hypoxic Preconditioning Attenuated Simulated Ischemia/Reperfusion Injury in Cultured Bone Marrow Stromal Cells. J Cytol Histol 5: 222. doi:10.4172/2157-7099.1000222

many stimuli, including cleaving DNA repair molecules, depredating the anti-apoptosis proteins, cleaving extra cellular matrix protein and other related molecules [27,28]. Wang et al reported that apoptosis suppression by HP correlated with the prevention of mitochondrial dysfunction and promotion of ERK and Akt phosphorylation in hypoxia and reoxygenation injury [29], and Akt signaling pathway was also found to play a critical role in the prevention of apoptotic cell death by inhibiting caspase release [21]. Moreover, the over expression of Bcl2 and maintenance of MMP in HP cells presumably involves in the protective effects [30,31]. In this study, the up-regulated expression of HIF-1a protected the BMSCs from subsequent I/R injury as evidenced by decreased caspase- 3 release and increased cell viability in HP groups, $8 \mathrm{~h} \mathrm{HP}$ represented a prominent role in antiapoptosis after I/R injury.

A proper HP time is also important for defending ischemia, prolonged HP failed to exert protective effects and even seemed deleterious in some cells [29]. In our system, sublethal hypoxia $2 \mathrm{~h}$ to $8 \mathrm{~h}$ before critical insult time-dependently attenuated apoptosis and resulted in discrepant cell viability, $8 \mathrm{~h}$-HP presented a distinguished activeness. The underlying mechanism needs further research.

In conclusion, HP could protect BMSCs from critical anoxia via up-regulating HIF-1a content and decreasing caspase-3 release and apoptosis in cultured BMSCs. A suitable HP time might develop a more considerable protection, which would produce a beneficial effect on cell therapy.

\section{References}

1. Chen J, Li Y, Wang L, Zhang Z, Lu D, et al. (2001) Therapeutic benefit of intravenous administration of bone marrow stromal cells after cerebral ischemia in rats. Stroke 32: 1005-1011.

2. Malgieri A, Kantzari E, Patrizi MP, Gambardella S (2010) Bone marrow and umbilical cord blood human mesenchymal stem cells: state of the art. Int J Clin Exp Med 3: 248-269.

3. Momin EN, Mohyeldin A, Zaidi HA, Vela G, Quiñones-Hinojosa A (2010) Mesenchymal stem cells: new approaches for the treatment of neurological diseases. Curr Stem Cell Res Ther 5: 326-344

4. Wei L, Fraser JL, Lu ZY, Hu X, Yu SP (2012) Transplantation of hypoxia preconditioned bone marrow mesenchymal stem cells enhances angiogenesis and neurogenesis after cerebral ischemia in rats. Neurobiol Dis 46: 635-645.

5. Hess DC, Borlongan CV (2008) Stem cells and neurological diseases. Cell Prolif 41: 94-114.

6. Sortwell CE, Pitzer MR, Collier TJ (2000) Time course of apoptotic cell death within mesencephalic cell suspension grafts: implications for improving grafted dopamine neuron survival. Exp Neurol 165: 268-277.

7. Haider H, Ashraf M (2008) Strategies to promote donor cell survival: combining preconditioning approach with stem cell transplantation. J Mol Cell Cardiol 45 554-566.

8. Mohyeldin A, Garzón-Muvdi T, Quiñones-Hinojosa A (2010) Oxygen in stem cell biology: a critical component of the stem cell niche. Cell Stem Cell 7: 150-161.

9. Wu LY, Ding AS, Zhao T, Ma ZM, Wang FZ, et al. (2005) Underlying mechanism of hypoxic preconditioning decreasing apoptosis induced by anoxia in cultured hippocampal neurons. Neurosignals 14: 109-116.

10. Deng W, Bivalacqua TJ, Chattergoon NN, Jeter JR, Jr, Kadowitz PJ (2004) Engineering ex vivo-expanded marrow stromal cells to secrete calcitonin generelated peptide using adenoviral vector. Stem Cells 22: 1279-1291.

11. Mangi AA, Noiseux N, Kong D, He H, Rezvani M, et al. (2003) Mesenchymal stem cells modified with Akt prevent remodeling and restore performance of infarcted hearts. Nat Med 9: 1195-1201.

12. Muscari C, Bonafé F, Stanic I, Flamigni F, Stefanelli C, et al. (2005) Polyamine depletion reduces TNFalpha/MG132-induced apoptosis in bone marrow stromal cells. Stem Cells 23: 983-991.

13. Wu LY, Ding AS, Zhao T, Ma ZM, Wang FZ, et al. (2004) Involvement of increased stability of mitochondrial membrane potential and overexpression of $\mathrm{Bcl}-2$ in enhanced anoxic tolerance induced by hypoxic preconditioning in cultured hypothalamic neurons. Brain Res 999: 149-154.

14. Liu M, Alkayed NJ (2005) Hypoxic preconditioning and tolerance via hypoxia inducible factor (HIF) 1alpha-linked induction of P450 2C11 epoxygenase in astrocytes. J Cereb Blood Flow Metab 25: 939-948.

15. Lu GW, Yu S, Li RH, Cui XY, Gao CY (2005) Hypoxic preconditioning: a novel intrinsic cytoprotective strategy. Mol Neurobiol 31: 255-271.

16. Hu X, Yu SP, Fraser JL, Lu Z, Ogle ME, et al. (2008) Transplantation of hypoxiapreconditioned mesenchymal stem cells improves infarcted heart function via enhanced survival of implanted cells and angiogenesis. J Thorac Cardiovasc Surg 135: 799-808.

17. Theus MH, Wei L, Cui L, Francis K, Hu X, et al. (2008) In vitro hypoxic preconditioning of embryonic stem cells as a strategy of promoting cell survival and functional benefits after transplantation into the ischemic rat brain. Exp Neurol 210: 656-670.

18. Ogle ME, Yu SP, Wei L (2009) Primed for lethal battle: a step forward to enhance the efficacy and efficiency of stem cell transplantation therapy. $J$ Thorac Cardiovasc Surg 138: 527.

19. Francis KR, Wei L (2010) Human embryonic stem cell neural differentiation and enhanced cell survival promoted by hypoxic preconditioning. Cell Death Dis 1: e22.

20. Hu X, Wei L, Taylor TM, Wei J, Zhou X, et al. (2011) Hypoxic preconditioning enhances bone marrow mesenchymal stem cell migration via Kv2.1 channe and FAK activation. Am J Physiol Cell Physiol 301: C362-372.

21. Uchiyama T, Engelman RM, Maulik N, Das DK (2004) Role of Akt signaling in mitochondrial survival pathway triggered by hypoxic preconditioning. Circulation 109: 3042-3049.

22. Gidday JM, Fitzgibbons JC, Shah AR, Park TS (1994) Neuroprotection from ischemic brain injury by hypoxic preconditioning in the neonatal rat. Neurosci Lett 168: 221-224.

23. Sharp FR, Ran R, Lu A, Tang Y, Strauss KI, et al. (2004) Hypoxic preconditioning protects against ischemic brain injury. NeuroRx 1: 26-35.

24. Taie S, Ono J, Iwanaga Y, Tomita S, Asaga T, et al. (2009) Hypoxia-inducible factor-1 alpha has a key role in hypoxic preconditioning. J Clin Neurosci 16 : 1056-1060.

25. Li L, Qu Y, Li J, Xiong Y, Mao M, et al. (2007) Relationship between HIF-1alpha expression and neuronal apoptosis in neonatal rats with hypoxia-ischemia brain injury. Brain Res 1180: 133-139.

26. Li Y, Xia ZL, Chen LB (2011) HIF-1- $\alpha$ and survivin involved in the anti-apoptotic effect of 2ME2 after global ischemia in rats. Neurol Res 33: 583-592.

27. Park SY, Cho SJ, Kwon HC, Lee KR, Rhee DK, et al. (2005) Caspaseindependent cell death by allicin in human epithelial carcinoma cells: involvement of PKA. Cancer Lett 224: 123-132.

28. Zuliani T, Obriot H, Tual M, Lachman-Weber N, Dumas M, et al. (2008) Variable Bax antigenicity is linked to keratinocyte position within epidermal strata and UV-induced apoptosis. Exp Dermatol 17: 125-132.

29. Wang JA, Chen TL, Jiang J, Shi H, Gui C, et al. (2008) Hypoxic preconditioning attenuates hypoxia/reoxygenation-induced apoptosis in mesenchymal stem cells. Acta Pharmacol Sin 29: 74-82.

30. Dispersyn G, Nuydens R, Connors R, Borgers M, Geerts H (1999) Bcl-2 protects against FCCP-induced apoptosis and mitochondrial membrane potential depolarization in PC12 cells. Biochim Biophys Acta 1428: 357-371.

31. Gabriel B, Sureau F, Casselyn M, Teissié J, Petit PX (2003) Retroactive pathway involving mitochondria in electroloaded cytochrome c-induced apoptosis. Protective properties of Bcl-2 and Bcl-XL. Exp Cell Res 289: 195-210. 